\title{
RESPOSTAS DA FREQUÊNCIA CARDÍACA AO EXERCÍCIO RESISTIDO E SUA RELAÇÃO COM A VARIABILIDADE DA FREQUÊNCIA CARDÍACA EM INDIVÍDUOS COM FATORES DE RISCO PARA DOENÇAS CARDIOVASCULARES
}

\author{
HEART RATE RESPONSES TO RESISTANCE EXERCISE AND ITS RELATION TO THE HEART RATE \\ VARIABILITY IN INDIVIDUALS WITH RISK FACTORS FOR CARDIOVASCULAR DISEASES
}

\author{
Leonardo Cazelato $^{\mathrm{a}^{*}}$, Pedro Henrique Rodrigues ${ }^{\mathrm{b}^{* *}}$, Robison José Quitério ${ }^{\mathrm{c}^{* * *}}$ \\ aleocazelato@yahoo.com.br, bpedrohr.edfisica@gmail.com, crobisonq@gmail.com \\ *Centro Universitário Toledo - Araçatuba (SP), Brasil \\ **Universidade de Marília - Marília (SP), Brasil \\ ***Universidade Estadual Paulista - Rio Claro (SP), Brasil
}

Data de recebimento do artigo: 25/04/2017

Data de aceite do artigo: 26/09/2017

\section{RESUMO}

Introduçáo: A ação dos ramos simpático e parassimpático do sistema nervoso autônomo (SNA) sobre o coração promove aumento e diminuiçáo, respectivamente, da frequência cardíaca (FC). A variação da FC na transição repouso-exercício pode indicar como se comporta o sistema nervoso parassimpático nos segundos iniciais do esforço, já que ocorre inibição da modulação vagal como resposta inicial da FC no exercício. Uma variabilidade da frequência cardíaca (VFC) reduzida na condição basal pode indicar adaptação ineficaz do SNA. Em indivíduos portadores de fatores de risco para doenças cardiovasculares, as respostas da FC durante o exercício levam mais tempo para atingir valores ideais, o que pode ser atribuído à modulação parassimpática ineficaz no início do esforço, porém não há evidência de que a função vagal prejudicada esteja relacionada à menor resposta da FC no início do exercício resistido. Objetivo: Avaliar a magnitude das respostas da FC durante o esforço máximo através do teste de uma repetição máxima (1RM) e verificar sua relação com o comportamento da VFC na condição basal em treze indivíduos, com idades entre 50 e 71 anos e portadores de fatores de risco para doenças cardiovasculares. Métodos: Foram registrados os valores de FC e dos intervalos RR na condição basal e em protocolo de teste de $1 \mathrm{RM}$ antes e durante o esforço. A partir dos dados obtidos, foram calculados a variaçáo da FC repousoexercício e os índices SD1 e RMSSD da VFC na condição basal. Resultados: Foi encontrada uma forte correlação positiva entre variação da FC repouso-exercício e índices da VFC na condição basal. Conclusão: Indivíduos com melhor modulação vagal na condição basal apresentam maior variação da FC no exercício resistido máximo.

Palavras-chave: Sistema nervoso autônomo; sistema nervoso parassimpático; frequência cardíaca; fatores de risco; doenças cardiovasculares.

\section{ABSTRACT}

Introduction: The action of the sympathetic and parasympathetic branches of the autonomic nervous system (ANS) on the heart causes increase and decrease, respectively, of heart rate (HR). The variation of $\mathrm{HR}$ in the rest to exercise transition may indicate how the parasympathetic nervous system behaves in the first seconds of physical exertion because an inhibition of vagal modulation occurs as an initial response of $\mathrm{HR}$ in the exercise. A reduced heart rate variability (HRV) in basal condition may indicate an inefficient adaptation of the ANS. In individuals that have risk factors for cardiovascular diseases, HR response during exercise take longer to reach ideal values, which may be attributed to inefficient parasympathetic modulation at the beginning of physical exertion, but there is no evidence that impaired vagal function is related to the lower HR response at the beginning of the resistance exercise. Objective: To evaluate the magnitude of HR responses during maximum physical exertion using the One Maximum Repetition Test 
(1RM) and to verify its relation with the behavior of HRV during the basal condition in thirteen people, aged 50-71 with risk factors for cardiovascular diseases. Methods: We recorded values of HR and RR intervals in basal condition and in the $1 \mathrm{RM}$ test protocol before and during the physical exertion. Based on the data we calculated the rest/exercise HR variation and the SD1 and RMSSD indexes of HRV in basal condition. Results: We found a strong positive correlation between rest/exercise HR variation and HRV indexes in basal condition. Conclusion: Individuals with better vagal modulation in basal condition show a greater variation of HR in maximum resistance exercise.

Keywords: Autonomic nervous system; parasympathetic nervous system; heart rate; risk factors; cardiovascular diseases.

\section{Introdução}

Ajustes rápidos no sistema cardiovascular são necessários durante o exercício físico para garantir os níveis necessários de oxigênio e nutrientes para o metabolismo muscular ${ }^{1,2}$. O coração possui um mecanismo intrínseco para o controle da frequência cardíaca (FC), que tem a função de gerar e propagar estímulos elétricos por todo músculo cardíaco garantindo seu funcionamento ${ }^{3}$. Entretanto, a FC é controlada principalmente pelo sistema nervoso autônomo (SNA), por meio das ramificaçóes simpáticas para todo o coração e das ramificaçóes parassimpáticas para regiáo do nodo sinusal, miocardio atrial e nodo atrioventricular $^{4}$. Em condições fisiológicas, o aumento da FC ocorre devido ao incremento da atividade simpática e diminuiçáo parassimpática, ao passo que um predomínio parassimpático é basicamente o motivo na redução da $\mathrm{FC}^{4-7}$.

Nos segundos iniciais da contração muscular, o aumento rápido da FC é atribuído à inibição da modulação vagal sobre o nodo sinusal ${ }^{2}$. Assim, tem sido constatado que durante o exercício físico ocorre aumento da FC, inicialmente por inibição vagal e, em seguida, por predomínio da modulação simpática, dependendo da intensidade e duração da contração muscular ${ }^{8}$.

Em um teste de esforço máximo de curta duração, como o teste de uma repetição máxima (1RM), a variação da FC na transição repouso-exercício pode fornecer informações importantes que não são observadas na condição basal, como a integridade do ramo parassimpático nos segundos iniciais do esforço, quando ocorre a retirada vagal e consequente ajustes cardiovasculares provenientes da modulação parassimpática ${ }^{9-11}$.

A variabilidade da frequência cardíaca (VFC), que pode ser definida como as mudanças no intervalo de tempo entre os batimentos cardíacos, também é um indicador da modulação autonômica cardíaca ${ }^{12}$. Uma VFC reduzida na condição basal pode ser indício de adaptação anormal e ineficaz do SNA, sinalizando um mau funcionamento desse sistema no indivíduo ${ }^{6,13}$.
A presença de fatores de risco para doenças cardiovasculares como hipertensão ${ }^{14-16}$, diabetes ${ }^{7,17}$, excesso de gordura corporal ${ }^{18-20}$, alteraçóes no perfil lipídico $^{21,22}$ e idade ${ }^{23,24}$ repercute na modulação autonômica cardíaca, já que as respostas da FC durante o exercício levam mais tempo para atingir os valores ideais $^{25,26}$, indicando modulação parassimpática insuficiente no início do esforço.

Essas repercussóes configuram-se como fortes preditores de risco cardiovascular, evidenciando a importância do estudo da FC e da sua variabilidade para uma avaliação da função autonômica mais prática e acessível, já que uma menor resposta da FC no teste de $1 \mathrm{RM}$ pode trazer indícios de comprometimento da modulação parassimpátca cardíaca. A hipótese deste estudo é que existe uma relação positiva entre a magnitude da variação da FC no teste de $1 \mathrm{RM}$ e a VFC na condição basal, o que facilita a identificação de desequilíbrios autonômicos e consequentes riscos ao sistema cardiovascular, considerando a maior praticidade e acessibilidade na observação da variação da FC em relação à análise da VFC.

Assim sendo, o objetivo desta pesquisa foi avaliar os ajustes da FC durante o teste de $1 \mathrm{RM}$ em indivíduos portadores de fatores de risco para doenças cardiovasculares e verificar se existe relaçáo entre a variação da $\mathrm{FC}$ na transição repouso-exercício e o comportamento da VFC na condição basal.

\section{Metodologia}

A amostra foi constituída por 13 voluntários, de ambos os sexos, com idades entre 50 e 71 anos e portadores de fatores de risco para doenças cardiovasculares (Tabela 1). Os voluntários foram submetidos a exames laboratoriais (colesterol total e frações, triglicerídeos, glicemia de jejum e hemograma) que tiveram finalidade diagnóstica, sendo que os critérios para inclusão no estudo foram: obesidade (IMC > que $\left.30 \mathrm{~kg} / \mathrm{m}^{2}\right)^{27}$; baixo nível de atividade física (classificados como sedentários ou irregularmente ativos 
segundo o International Physical Activity Questionnaire - IPAQ ${ }^{28}$; diabetes (glicemia $\left.>126 \mathrm{mg} / \mathrm{dL}\right)^{29}$; colesterol total $(\mathrm{CT})>200 \mathrm{mg} / \mathrm{dL}$; colesterol-lipoproteína de alta densidade (HDL-c) $<60 \mathrm{mg} / \mathrm{dL}$; colesterol-lipoproteína de baixa densidade (LDL-c) > $130 \mathrm{mg} / \mathrm{dL}$; triglicerídeos $(\mathrm{TG})>150 \mathrm{mg} / \mathrm{dL}^{30}$; pressão arterial sistêmica sistólica $>140 \mathrm{mmHg}$; e pressão arterial sistêmica diastólica $>90 \mathrm{mmHg}^{31}$.

Foram excluídos os voluntários praticantes de exercícios resistidos nos seis meses antecedentes ao estudo e os que apresentaram limitaçóes motoras que comprometessem a execução dos testes físicos, distúrbios respiratórios e distúrbios endócrinos. Todos os voluntários foram informados sobre os procedimentos experimentais e o caráter náo invasivo dos testes. Após terem lido e concordado, assinaram um termo de consentimento livre e esclarecido. $\mathrm{O}$ projeto (n. 1106/2014) foi submetido e aprovado pelo Comitê de Ética em Pesquisa Envolvendo Seres Humanos da Faculdade de Filosofia e Ciências da Universidade Estadual Paulista (Unesp), de Marília, de acordo com a Resoluçáo 196/96 do Conselho Nacional de Saúde e suas complementares. Foram registrados os dados de $\mathrm{FC}$ dos voluntários na condiçáo basal e no teste de 1RM no aparelho supino vertical, que tinha como objetivo determinar a força máxima dos indivíduos para o exercício de adução horizontal do ombro.

Os experimentos foram realizados sempre no mesmo período do dia (tarde), para padronizar as influências das variaçóes circadianas sobre o organismo. A sala experimental foi mantida em $24+2^{\circ} \mathrm{C}$, e a umidade relativa do ar, em $50+10 \%$. Para reduzir a ansiedade e expectativa por parte dos voluntários, foram feitos procedimentos de familiarização deles com o protocolo de teste de $1 \mathrm{RM}$, com o avaliador e com equipamentos.

O protocolo de testes foi aplicado em dois dias, com intervalo de 48 horas. No primeiro dia de experimento foram realizados anamnese (coleta dos dados pessoais, hábitos de vida e IPAQ), antropometria (peso corporal e estatura), registro da FC e dos intervalos $R R$ (iR-R) na condição basal e familiarização ao teste de $1 \mathrm{RM}$. No segundo dia de experimento foi realizado somente o teste de $1 \mathrm{RM}$.

Os voluntários realizaram os testes com roupas confortáveis, não ingeriram bebidas alcoólicas e/ou estimulantes e nem realizaram atividades físicas extenuantes nas 24 horas precedentes aos testes. No dia da realização dos testes, as condiçôes relacionadas ao estado de saúde do voluntário foram observadas, para verificar a ocorrência de uma noite de sono regular e para confirmar se as variáveis FC e PA estavam dentro dos limites de normalidade.

\section{Variabilidade da frequência cardíaca}

A FC e os iR-R instantâneos foram gravados durante o protocolo por um sistema digital de telemetria que consiste em um transmissor posicionado na altura do processo xifóide do sujeito e um monitor/receptor (Polar RS800 CX, Polar Electro Oy, Kempele, Finland). O sistema detecta a despolarizaçáo ventricular, que corresponde à onda $\mathrm{R}$ do eletrocardiograma, com frequência amostral de $1000 \mathrm{~Hz}$ e resolução temporal de um milissegundo (ms), sendo validado previamente ${ }^{32-34}$.

Inicialmente o sujeito foi posicionado na postura sentada e permaneceu em repouso, com o mínimo de movimentos e sem conversar, até que as variáveis fisiológicas se estabilizassem. Na sequência, foi feito o registro da FC com respiração espontânea durante 20 minutos.

Os dados foram transmitidos para um computador e filtrados utilizando-se o software Polar Precision Performance (versão 3.02.007). Em seguida, os dados foram analisados no software Kubios HRV (versão 2.0, University of Kuopio, Finland).

No domínio do tempo foram feitos cálculos estatísticos para obtenção da média dos valores instantâneos de FC em batimentos por minuto (bat/min), média dos iR-R em ms e raiz quadrada da somatória do quadrado das diferenças entre os $\mathrm{iR}-\mathrm{R}$ em ms em registro dividida pelo número dos iR-R em ms em um tempo determinado menos um (RMSSD) expresso em ms, que é representativo da modulação parassimpática ${ }^{6,12,13}$.

A análise das propriedades não lineares da VFC foi feita através da análise do plot de Poincaré, que é um mapa de pontos em coordenadas cartesianas, onde cada ponto é representado, no eixo horizontal X (abcissa), pelo iR-R normal precedente e, no eixo vertical Y (ordenada), pelo iR-R seguinte. $O$ desvio padráo dos pontos perpendiculares e ao longo da linha de identidade dá origem aos índices SD1 e SD2, respectivamente. Na análise não linear dos resultados, foi utilizado o índice SD1 que mede o desvio-padrão das distâncias dos pontos à diagonal $y=x$ e está relacionado com variabilidade em curto prazo, representando a atividade parassimpática ${ }^{35}$.

\section{Teste de $1 R M$}

O teste de 1RM foi realizado seguindo as recomendaçôes do American College of Sports Medicine (ACSM) $)^{36}$ no aparelho supino vertical, cujo movimento articular é a adução horizontal do ombro. Previamente ao teste, 
foi realizado um aquecimento constituído por: cinco minutos de exercício aeróbio em esteira ergométrica com intensidade em $50 \%$ da FC de reserva calculada pela estimativa da FC máxima (220-idade) - FC de repouso; aplicação da fórmula (FC de reserva $x$ intensidade $(50 \%)+\mathrm{FC}$ de repouso ${ }^{36}$; duas séries de 15 segundos de alongamento leve para o grupo muscular a ser testado; e dez repetiçóes do exercício sem carga. $\mathrm{O}$ teste foi iniciado cinco minutos após o aquecimento.

Para favorecer a detecção da FC pico (maior valor da FC observado antes do final da contração) no final do esforço, o teste de 1RM foi sempre iniciado na fase excêntrica do exercício, mesmo que houvesse necessidade de auxílio do avaliador para manutençáo do aparelho na posição inicial; não foi permitida uma pausa maior que um segundo entre as fases excêntrica e concêntrica.

Para encontrar a carga máxima para uma repetição, a resistência era aumentada progressivamente entre 2 a $10 \mathrm{~kg}$ até que o voluntário não conseguisse mais completar a tentativa subsequente, e quando isso ocorria, era subtraído $50 \%$ da carga acrescida na última tentativa. A carga inicial foi estimada de acordo com a carga utilizada no procedimento de familiarizaçáo. Foram realizadas, no máximo, cinco tentativas por sessão (dia), e entre as tentativas eram concedidos intervalos recuperativos de quatro minutos. Caso não fosse possível detectar a carga máxima para uma repetição nas cinco tentativas, o voluntário deveria voltar após 48 horas para realizar o teste novamente.

Inicialmente os indivíduos permaneceram em repouso na postura do teste até que FC se estabilizasse. Em seguida, a FC e os iR-R foram registrados durante 65 segundos em repouso pré-contração e durante as contraçóes musculares, utilizando-se o sistema digital de telemetria ${ }^{32,33,34}$ descrito anteriormente. Durante o teste foi mantida a respiração espontânea, sem apneia.

A partir dos dados de FC e dos iR-R foram calculados a FC de repouso (média da FC obtida nos 30 segundos de repouso precedentes ao teste) e a variaçáo da FC repouso/exercício (diferença entre a FC pico e FC de repouso).

\section{Análise estatística}

Os dados foram organizados sob a forma de estatística descritiva, com valores de média e desvio padrão. As variáveis categóricas foram descritas sob a forma de distribuição de frequência relativa e absoluta. A distribuição de normalidade foi verificada pelo teste de ShapiroWilk com correção pelo teste de Lillifors. Para analisar a relação entre a variação da $\mathrm{FC}$ repouso-exercício e os valores dos índices de VFC na condição basal foi realizado o teste de correlação de Pearson, sendo que quando o coeficiente de correlação de Pearson fosse menor que 0,30 , indicava-se fraca correlação, de 0,30 a 0,70 , correlaçáo moderada, e maior que 0,70 , forte correlaçáo. O nível de significância adotado foi de $5 \%$. Os dados foram analisados no software SPSS, versão 20.0 para Windows.

\section{Resultados}

$\mathrm{Na}$ Tabela 1, estão apresentados os dados referentes às características dos voluntários, os fatores de risco para doenças cardiovasculares presentes e os medicamentos em uso.

Tabela 1: Características demográficas e antropométricas, fatores de risco e medicamentos da amostra estudada

\begin{tabular}{lc} 
Características & $\mathbf{n}=\mathbf{1 3}$ \\
\hline Idade (anos) & $61,62 \pm 7,29$ \\
\hline Peso corporal $(\mathrm{kg})$ & $74,95 \pm 21,45$ \\
\hline IMC $\left(\mathrm{kg} / \mathrm{m}^{2}\right)$ & $29,45 \pm 7,28$ \\
\hline Fatores de risco & \\
\hline Diabéticos & $5(38,46 \%)$ \\
Hipertensos & $9(69,23 \%)$ \\
Obesos & $5(38,46 \%)$ \\
Dislipidêmicos & $9(69,23 \%)$ \\
Sedentários & $3(23,08 \%)$ \\
Medicamentos & \\
Hipoglicemiantes & $3(23,08 \%)$ \\
Vasodilatadores & $9(69,23 \%)$ \\
Betabloqueadores & $4(30,77 \%)$ \\
Diuréticos & $2(15,38 \%)$ \\
Anticoagulantes & $6(46,15 \%)$ \\
Antilipêmicos & $7(53,85 \%)$ \\
\hline
\end{tabular}

Fonte: Elaborada pelos autores, 2017.

IMC = Índice de massa corporal.

Os voluntários apresentaram variação média de $21,1 \pm 9,3 \mathrm{bat} / \mathrm{min}$ na transição repouso-exercício durante o teste de $1 \mathrm{RM}$, no qual a FC, que no repouso pré-esforço era de $81 \pm 10,9 \mathrm{bat} / \mathrm{min}$, atingiu $102,1 \pm 12,1$ $\mathrm{bat} / \mathrm{min}$ no pico do esforço. Foi encontrada uma forte correlação positiva entre a variação da $\mathrm{FC}$ repouso-exercício e índices da VFC obtidos na condição basal, que tiveram valores médios de $19,8 \pm 13,7$ ms para RMSSD e 14,0 $\pm 9,7$ para SD1. Esses dados são demonstrados nas Figuras 1 e 2. 
Figura 1: Dados da análise de correlação entre os ajustes da FC no esforço e RMSSD na condição basal

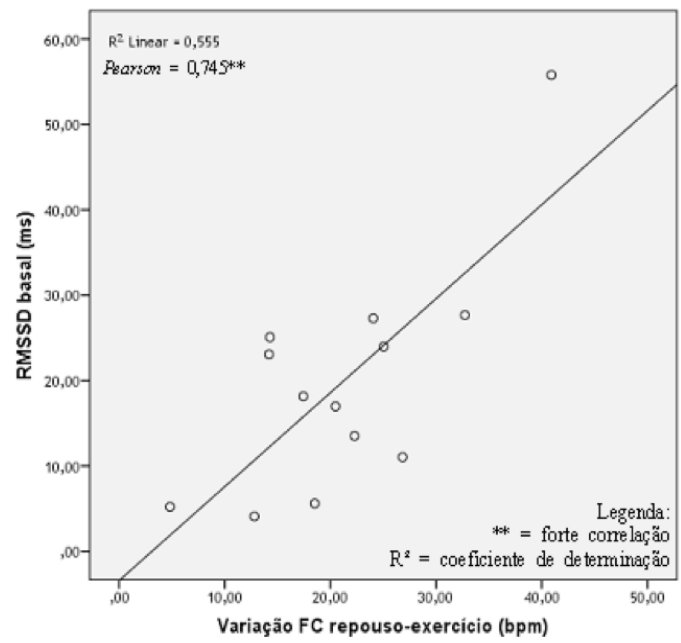

Fonte: Elaborada pelos autores, 2017.

Figura 2: Dados da análise de correlação entre os ajustes da FC no esforço e SD1 na condição basal

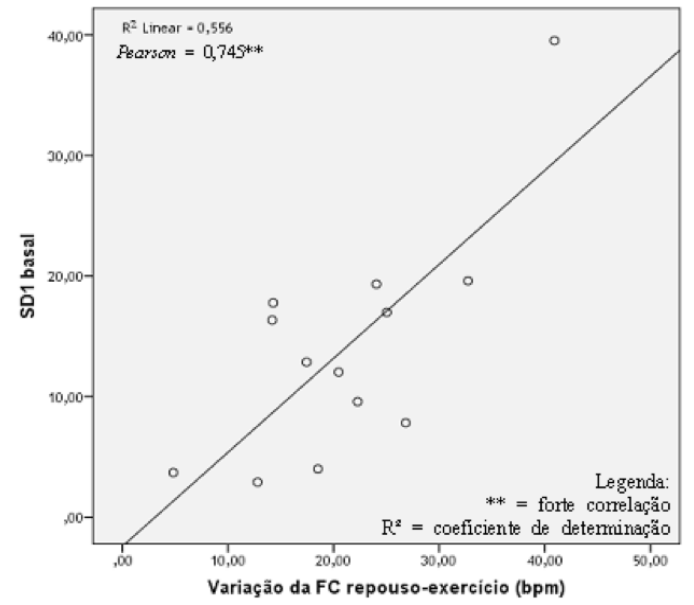

Fonte: Elaborada pelos autores, 2017.

\section{Discussão}

Este estudo investigou as respostas da FC em teste de 1RM no supino vertical em indivíduos com fatores de risco para doenças cardiovasculares. Não há na literatura valores de normalidade para variação da FC na transiçâoo repouso-exercício, porém alguns estudos investigaram essa resposta em diferentes populaçóes e método ${ }^{37,38}$. O principal resultado encontrado neste estudo é a variação da FC verificada na transição repouso-exercício estar associada com a modulação parassimpática basal.
O valor médio da FC em repouso da amostra (81 bat/ min) está dentro das faixas esperadas para a idade adulta, que vai de $50 \mathrm{bat} / \mathrm{min}$ a $100 \mathrm{bat} / \mathrm{min}^{39}$. Em relação aos índices de VFC representativos da modulaçáo parassimpática, Freeman et al. ${ }^{2}$ estabeleceram como normal para RMSSD na condição basal o valor de $27 \mathrm{~ms} \pm 12$, o que está acima do valor obtido neste estudo (19,8 ms). Não são encontrados na literatura valores de normalidade para o índice SD1. A provável causa dos baixos valores de VFC em repouso é a presença de fatores de risco para doenças cardiovasculares na amostra, já que é bem evidenciada a ocorrência de disfunção autonômica e consequente redução no componente vagal cardíaco nesta população ${ }^{14,26,40}$.

Para avaliação das respostas da FC ao esforço máximo, utilizamos o teste de 1RM, que teve um esforço com duração média de oito segundos, caracterizando um estímulo dependente essencialmente da inibição do sistema parassimpático sobre o nodo sinusal ${ }^{2,11,41,42}$. A variaçáo da FC repouso-exercício obtida neste estudo $(21,1$ $\mathrm{bat} / \mathrm{min}$ ) apresenta semelhança aos achados do estudo de Farinatti e Assis ${ }^{37}$, que, ao avaliar jovens de ambos os sexos, encontraram uma variação de $22,1 \mathrm{bat} / \mathrm{min}$, sendo que a FC variou de 74,9 bat $/ \mathrm{min}$ na condição de repouso para $97 \mathrm{bat} / \mathrm{min}$ no pico do esforço. Por outro lado, diferenciam-se dos achados de Beltrame et al. ${ }^{38}$, que observaram em indivíduos de meia idade uma variação de $11 \mathrm{bat} / \mathrm{min}$ nos 10 segundos iniciais de exercício intenso em cicloergômetro, ao passo que em jovens a variação foi de $19 \mathrm{bat} / \mathrm{min}$.

Com base nos estudos citados, não é possível afirmar que a idade por si só está relacionada com menores variações da FC no início do esforço, tampouco que a presença de fatores de risco para doenças cardiovasculares causa redução na magnitude da resposta da FC. No entanto, nos estudos que investigaram as respostas da FC no exercício resistido, os resultados foram bastante similares, podendo ser o tipo de exercício um fator determinante nos ajustes da FC na transiçâo repouso-exercício.

O principal resultado deste estudo foi a forte correlação positiva entre os índices RMSSD e SD1 da VFC na condição basal e a variação da FC durante o exercício, ou seja, indivíduos com funçáo vagal prejudicada apresentaram menor variação da FC no esforço resistido máximo de curta duração, explicada por uma retirada vagal menos eficiente.

Não há na literatura estudo que fez o mesmo tipo de análise entre essas duas variáveis para este tipo de exercício. No entanto, Ricardo et al..$^{43}$ mostraram, em protocolo de teste com exercício em cicloergômetro, que há maiores respostas da FC na transição repouso-exercício em indivíduos melhores condicionados aerobiamente, o que indiretamente indica maior eficiência vagal entre os indivíduos mais condicionados. Os autores ainda 
complementaram, sugerindo que a variaçáo da FC na transição repouso-exercício pode fornecer um relevante indicador prognóstico, relativo à integridade vagal cardíaca. Apesar da importante afinidade entre os resultados, deve-se ressaltar que o estudo citado utilizou um cicloergômetro no protocolo de teste, diferenciando-se do teste de 1RM utilizado neste estudo.

Algumas limitaçóes devem ser consideradas neste estudo, como a ausência de um grupo controle, a não homogeneidade da amostra em relação ao uso de medicamentos, especialmente beta-bloqueadores, que interferem no controle autonômico cardíaco ${ }^{44}$, além dos distintos fatores de risco presentes, o que provavelmente influencia nas respostas da FC durante o esforço ${ }^{26,45}$. Devido às interaçóes de causa e efeito entre si, os fatores de risco para doenças cardiovasculares geralmente são concomitantes ${ }^{46,47}$, dificultando a formação de um grupo homogêneo, isto é, com apenas um fator de risco, ou que utiliza apenas um tipo de medicamento.

A contribuição do estudo está em avaliar as respostas cardiovasculares ao exercício físico em uma população com diferentes tipos de fatores de risco e que usava diversas combinaçôes de medicamentos, ou seja, condiçôes reais encontradas em centros de prescrição de exercício resistido, como academias, clubes e clínicas. Desta forma, este estudo buscou compreender melhor o efeito agudo do exercício resistido máximo de curta duração sobre a FC durante o esforço e facilitar a identificação de possíveis desequilíbrios autonômicos, especificamente da modulação parassimpática para essa população.

\section{Conclusão}

A partir dos resultados obtidos neste estudo com indivíduos portadores de fatores de risco para doenças cardiovasculares, conclui-se que a variação da $\mathrm{FC}$ ao exercício resistido máximo está diretamente relacionada com modulação parassimpática de repouso, ou seja, indivíduos com melhor modulação vagal apresentam maior variação da $\mathrm{FC}$ ao exercício resistido máximo.

\section{Referências}

1. Williamson JW, Fadel PJ, Mitchell JH. New insights into central cardiovascular control during exercise in humans: a central command update. Exp Physiol. 2006;91(1):51-8.

2. Freeman JV, Dewey FE, Hadley DM, Myers J, Froelicher VF. Autonomic nervous system interaction with the cardiovascular system during exercise. Prog Cardiovasc Dis. 2006;48(5):342-62.
3. Guyton AC, Hall JE. Tratado de fisiologia médica. 10. ed. Rio de Janeiro: Guanabara Koogan; 2002.

4. Aubert AE, Seps B, Beckers F. Heart rate variability in athletes. Sports Med. 2003;33(12):889-919.

5. Paschoal MA, Petrelluzzi KFS, Gonçalves NVO. Controle autonômico cardíaco durante a execução de atividade física dinâmica de baixa intensidade. Rev Soc Cardiol. 2003;13(5):1-11.

6. Rajendra Acharya U, Paul Joseph K, Kannathal N, Lim CM, Suri JS. Heart rate variability: a review. Med Biol Eng Comput. 2006;44(12):1031-51.

7. Rolim LCSP, Sá JR, Chacra AR, Dib SA. Neuropatia autonômica cardiovascular diabética: fatores de risco, impacto clínico e diagnóstico precoce. Arq Bras Cardiol. 2008;90(4):24-32.

8. Takahashi AC, Melo RC, Quitério RJ, Silva E, Catai AM. The effect of eccentric strength training on heart rate and on its variability during isometric exercise in healthy older men. Eur J Appl Physiol. 2009;105(2):315-23.

9. Almeida MB, Ricardo DR, Araújo CGS. Variabilidade da frequência cardíaca em um teste de exercício verdadeiramente máximo. Rev Socerj. 2005;18(6):534-41.

10. Da Paschoa DC, Coutinho JFS, Almeida MB. Análise da variabilidade da frequência cardíaca no exercício de força. Rev Socerj. 2006;19(5):385-90.

11. Mitchell JH. Neural control of the circulation during exercise: insights from the 1970-1971 Oxford studies. Exp Physiol. 2012;97(1):14-9.

12. Task Force of the European Society of Cardiology and the North American Society of Pacing Electrophysiology. Heart rate variability: standards of measurement, physiological interpretation and clinical use. Circulation. 1996;93(5):1043-65.

13. Vanderlei LCM, Pastre CM, Hoshi RA, Carvalho TD, Godoy MF. Noçóes básicas de variabilidade da frequência cardíaca e sua aplicabilidade clínica. Rev Bras Circ Cardiovasc. 2009;24(2):205-17.

14. Thayer JF, Yamamoto SS, Brosschot JF. The relationship of autonomic imbalance, heart rate variability and cardiovascular disease risk factors. Int J Cardiol. 2010;141(2):122-31.

15. Michelini LC. Regulação momento a momento da pressão arterial na normotensão e hipertensão: implicaçôes fisiopatológicas. Hipertensão. 2000;3(3):90-8.

16. Barbosa Filho J, Barbosa PRB, Cordovil I. Modulação autonômica do coração na hipertensão arterial sistêmica. Arq Bras Cardiol. 2002;78(2):181-8.

17. Vinik AI, Maser RE, Mitchell BD, Freeman R. Diabetic autonomic neuropathy. Diabetes Care. 2003;26(5):1553-79.

18. Rissanen P, Franssila-Kallunki A, Rissanen A. Cardiac parasympathetic activity is increased by weight loss in health obese women. Obes Res. 2001;9(10):637-43. 
19. Laederach-Hofmann $\mathrm{K}$, Mussgay L, Rúddel $\mathrm{H}$. Autonomic cardiovascular regulation in obesity. J Endocrinol. 2000;164(1):59-66.

20. Lopes HF, Egan BM. Desequilíbrio autonômico e síndrome metabólica: parceiros patológicos em uma pandemia global emergente. Arq Bras Cardiol. 2006;87(4):538-47.

21. Liao D, Sloan RP, Cascio WE, Folsom AR, Liese AD, Evans GW, et al. Multiple metabolic syndrome is associated with lower heart rate variability. Diabetes Care. 1998;21(12):2116-28.

22. Kimura T, Matsumoto T, Akiyoshi M, Owa $\mathrm{Y}$, Miyasaka N, Aso T, et al. Body fat and blood lipids in postmenopausal women are relating to reting autonomic nervous system activity. Eur J Appl Physiol. 2006;97(5):542-7.

23. Lopes FL, Pereira FM, Reboredo MM, Castro TM, Vianna JM, Novo Júnior JM, et al. Redução da variabilidade da frequência cardíaca em indivíduos de meia-idade e o efeito do treinamento de força. Rev Bras Fisioter. 2007;11(2):113-9.

24. De Meersman RE, Stein PK. Vagal modulation and aging. Biol Psychol. 2007;74(2):165-73.

25. Moreira SR, Simóes GC, Hyane WC, Campbell CSG, Simões HG. Identificação do limiar anaeróbio em indivíduos com diabetes tipo-2 sedentários e fisicamente ativos. Rev Bras Fisioter. 2007;11(4):289-96.

26. Tibana RA, Boullosa DA, Leicht AS, Prestes J. Women with metabolic syndrome present different autonomic modulation and blood pressure response to an acute resistance exercise session compared with women without metabolic syndrome. Clin Physiol Funct Imaging. 2013;33(5):364-72.

27. World Health Organization. Obesity: preventing and managing the global epidemic. Report of a World Health Organization Consultation. Geneva: WHO; 2000.

28. Matsudo S, Araújo T., Matsudo V, Andrade D, Andrade E, Oliveira LC, et al. Questionário Internacional de Atividade Física (IPAQ): estudo de validade e reprodutibilidade no Brasil. Rev Bras Ativ Fís Saúde. 2001;6(2):5-18.

29. Sociedade Brasileira de Diabetes. Diretrizes da Sociedade Brasileira de Diabetes. 3a ed. Itapevi: A. Araújo Silva Farmacêutica; 2009.

30. Xavier H. T., Izar M. C., Faria Neto J. R., Assad M. H., Rocha V. Z., Sposito A. C., et al. V Diretriz Brasileira de Dislipidemias e Prevenção a Aterosclerose. Arq Bras Cardiol. 2013;101(4 Supl 1):1-2.

31. Sociedade Brasileira de Cardiologia, Sociedade Brasileira de Hipertensão, Sociedade Brasileira de Nefrologia. VI Diretrizes Brasileiras de Hipertensão Arterial. Arq Bras Cardiol. 2010;95(1 Supl 1):1-51.

32. Loimaala A, Sievänen H, Laukkanen R, Pärkkä J, Vuori I, Huikuri H. Accuracy of a novel real-time microprocessor QRS detector for heart rate variability assessment. Clin Physiol. 1999;19(1):84-8.
33. Gamelin FX, Berthoin S, Bosquet L. Validity of the polar S810 heart rate monitor to measure R-R intervals at rest. Med Sci Sports Exerc. 2006;38(5):887-93.

34. Rezende Barbosa MPC, Silva NT, Azevedo FM, Pastre CM, Vanderlei LC. Comparison of Polar RS800G3 heart rate monitor with Polar S810i and electrocardiogram to obtain the series of RR intervals and analysis of heart rate variability at rest. Clin Physiol Funct Imaging. 2016;36(2):112-7.

35. Brennan M, Palaniswami M, Kamen P. Do existing measures of Poincaré plot geometry reflect nonlinear features of heart rate variability. IEEE Trans Biomed Eng. 2001;48(11):1342-7.

36. American College of Sports Medicine. Diretrizes do ACSM para os testes de esforço e sua prescrição. Rio de Janeiro: Guanabara Koogan; 2010.

37. Farinatti PTV, Assis BFCB. Estudo da frequência cardíaca, pressão arterial e duplo-produto em exercícios contra-resistência e aeróbio contínuo. Rev Bras Ativ Fís Saúde. 2000;5(2):5-16.

38. Beltrame T, Karsten M, Chacon-Mikahil MPT, Madruga VA, Silva E, Borghi-Silva A, et al. Influência da idade no comportamento da frequência cardíaca na transição repouso-exercício: uma análise por deltas e regressão linear. Rev Bras Med Esporte. 2012;18(5):300-4.

39. Sociedade Brasileira de Cardiologia. Diretrizes da Sociedade Brasileira de Cardiologia sobre análise e emissão de laudos eletrocardiográficos (2009). Arq Bras Cardiol. 2009;93(3 Supl 2):1-19.

40. Sevre K, Lefrandt JD, Nordby G, Os I, Mulder M, Gans RO, et al. Autonomic function in hypertensive and normotensive subjects: the importance of gender. Hypertension. 2001;37(6):1351-6.

41. Quitério RJ, Moraes FR, Oliveira L, Teixeira LC, Gallo Junior L, Catai AM, et al. Influences of torque and joint angle on heart rate responses during isometric exercise in young men. Rev Bras Fisioter. 2007;11(3):185-90.

42. Quitério RJ, Melo RC, Takahashi ACM, Aniceto IAV, Silva E. Catai AM. Torque, myoeletric sygnal and heart rate responses during concentric and eccentric exercises in older men. Rev Bras Fisioter. 2011;15(1):8-14.

43. Ricardo DR, Almeida MB, Franklin BA, Araújo CG. Initial and final exercise heart rate transients: influence of gender, aerobic fitness and clinical status. Chest. 2005;127(1):318-27.

44. Miguel FM, Grings LA, Pereira GB, Leite RD, Vieira A, Sousa NMF, et al. Different cardiovascular responses to a resistance training session in hypertensive women receiving propanolol compared with normotensive controls. Sci World J. 2012;2012:913271.

45. Ichihara Y, Ohno J. Suzuki M, Anno T, Sugino M, Nagata K. Blunt circulatory response to exercise in coronary high-risk subjects among apparently healthy Japanese. Circ J. 2004;68(4):286-93. 
46. Ferreira CCC, Peixoto MRG, Barbosa MA, Silveira ÉA. Prevalência de fatores de risco cardiovascular em idosos usuários do Sistema Único de Saúde de Goiânia. Arq Bras Cardiol. 2010;95(5):621-8.
47. Petersen LC, Chinazzo H., Saldanha C, Basso M, Garcia P, Bartholomay, et al. Fatores de risco cardiovasculares e comorbidades em ambulatórios de cardiologia da região metropolitana de Porto Alegre, RS. Rev Amrigs. 2011;55(3):217-23.

Como citar este artigo:

Cazelato L, Rodrigues PH, Quitério RJ. Respostas da frequência cardíaca ao exercício resistido e sua relação com a variabilidade da frequência cardíaca em indivíduos com fatores de risco para doenças cardiovasculares. Rev. Aten. Saúde. 2018;16(55):21-28. 